\title{
Contribution-Factor based Fuzzy Min-Max Neural Network: Order-Dependent Clustering for Fuzzy System Identification
}

\author{
Peixin Hou ${ }^{1}$, Jiguang Yue ${ }^{1}$, Hao Deng ${ }^{2}$, Shuguang Liu ${ }^{3}$, Qiang Sun ${ }^{1}$ \\ ${ }^{1}$ Department of Control Science and Engineering, Tongji University, Shanghai, China \\ E-mails: \{houpeixin, yuejiguang, 10qsun\}@tongji.edu.cn \\ ${ }^{2}$ School of Physics and Electronics, Henan University, Kaifeng, China \\ E-mail: gavind@163.com \\ ${ }^{3}$ Department of Hydraulic Engineering, Tongji University, Shanghai, China \\ E-mail:liusgliu@tongji.edu.cn
}

Received 19 May 2017

Accepted 3 March 2018

\begin{abstract}
This study addresses the construction of Takagi-Sugeno-Kang (TSK) fuzzy models by means of clustering. A contribution-factor based fuzzy min-max neural network (CFMN) is developed based on Simpson's well-known fuzzy min-max neural network (FMNN) for clustering. The contribution-factor (CF) is also known as the typical pattern, and the membership threshold above which a pattern can be a CF of a cluster can be specified by the user. The stability issue is addressed and unnecessary overlaps in FMNN can be avoided. Furthermore, two considerations are combined in the clustering process to fully exploit the information in the data: 1) patterns (points) are generated in a sequence, so it's reasonable to capture the order-dependent information of data, and 2) the clustering process shouldn't be influenced too much by noisy data or outliers. As a result, CFMN can put most cluster centers in high-density regions of clusters without influence of the low-density regions. This feature is very important when clusters are used as fuzzy rules because the high-density region of some cluster can be interpreted as the most common part of that rule. Simulations are performed to illustrate the clustering behavior of CFMN and identification performance of the resulting fuzzy inference system (CFMN-FIS). It is shown that the proposed algorithm is fast to learn and has good prediction performance.
\end{abstract}

Keywords: fuzzy min-max neural network, clustering, adaptive resonance theory, contribution-factor, order-dependent, fuzzy inference system

\section{Introduction}

Fuzzy identification is an effective tool for the approximation of uncertain nonlinear systems on the basis of measured data. The data driven construction of fuzzy models has become an important topic of research with a wide range of real-world applications. The Takagi-Sugeno-Kang (TSK) ${ }^{1}$ model is a method of fuzzy inference well known in the fuzzy systems literature. The great advantage of the TSK model is its representative power. It is capable of describing a highly nonlinear system using a small number of rules ${ }^{2}$. Generally, fuzzy system identification consists of structure identification and parameter learning. Structure identification is used to determine the fuzzy membership functions and the appropriate number of rules. Parameter learning concerns the calculation of coefficients of each rule. 
As to the TSK model, the structure identification is based on a fuzzy partition of the input space and the parameter learning can be performed by standard linear least-squares methods when the premise parameters are fixed.

One of the most common approaches to structure identification is the utilization of clustering analysis. As defined in Ref. 3, the goal of clustering analysis is to discover natural groups of a set of points according to intrinsic characteristics or similarity. In other words, clustering divides data into groups, in such a manner that similar instances are grouped in the same group, producing a concise representation of a system's behavior ${ }^{4}$. Moreover, groups can be used to establish some hypothesis about the structure present in the data. Compared with other classical methods of membership functions identification that are complex or computationally demanding, for example, gradient descent ${ }^{5}$, genetic algorithms ${ }^{6}$, and Kalman filtering ${ }^{7}$, clustering can be used to make groups of related data and reduce the complexity of the model when we have a lot of data and we have no more information about it ${ }^{8}$.

Although many clustering algorithms have been used for fuzzy modeling ${ }^{491011}$, there are two considerations that have not been combined to fully exploit the information in the data.

1. Most of the clustering algorithms proposed in the literature didn't consider the orderdependent information. As the patterns (points) are generated by the underlying system in a sequence, not in a bunch, it's reasonable to capture the order-dependent information of data. In fuzzy system identification, the rule can be seen as a cluster in the feature space, thus if the clusters can adapt according to the information accounting for pattern generation order, we will get more informative rules.

2. Uncertainty in the data should be considered. That is, the clustering process shouldn't be influenced too much by noisy data or outliers.

This motivates us to use the fuzzy min-max clustering neural network (FMNN) ${ }^{12}$ with conceptually simple but powerful on-line learning process ${ }^{13}$. The network is order-dependent in the sense that the clustering result may be different if the input patterns are presented to the algorithm in a different order. However, FMNN doesn't consider the noise contained in patterns and tries to include all patterns in hyperboxes (clusters). This makes the network very unstable and the clusters generated are very close to each other.

In this paper, the contribution-factor based fuzzy min-max neural network (CFMN) is developed based on FMNN to explicitly exploit the orderdependent information in the clustering process and incorporates significant modifications that improve the noise-resistant capability of FMNN. The contribution-factor (CF) is also known as the typical pattern, and the membership threshold above which a pattern can be a CF of a cluster can be specified by the user. CFMN also introduces a parameter that specifies the minimum inter-cluster distance to ensure well-separated clusters. Furthermore, CFMN can put most cluster centers in the high-density region of clusters without influence of low-density regions. This feature is very important when clusters are used as fuzzy rules because the high-density region of some cluster can be interpreted as the most common part of that rule. Performance of the resulting fuzzy inference system (CFMN-FIS) is demonstrated on two nonlinear benchmark processes and three real-world data sets widely used in the fuzzy modeling literature. The obtained results are compared with results from the literature. It is shown that the proposed algorithm is fast to learn and has good prediction performance.

The main contributions are summarized as follows:

1. This study shows that the data-order information can be exploited in the clustering process. This information helps to discover the highdensity region of clusters.

2. We find that the learning process of FMNN has some kind of periodicity, which significantly impacts on the stability performance.

3. The proposed CFMN is intuitive in parameter choosing. The user can easily specify the 
cluster size and inter-cluster distance according to different situations.

4. CFMN has two advantages over existing clustering algorithms. Firstly, the estimated clusters are not affected by the clusters which the algorithm fails to discover. Secondly, the high-density region of a cluster is enclosed by a hyperbox without influence of low-density regions. These two features are essential to generate more meaningful fuzzy rules for the TSK-type CFMN-FIS.

The rest of this paper is organized as follows. Section 2 analyses the stability issue of FMNN. In Section 3, a detailed description of CFMN is given. Section 4 describes the resulting fuzzy model. Section 5 illustrates the feature of CFMN. The comparison of CFMN-FIS with various algorithms are also given. Finally, conclusions are given in Section 6.

\section{Analysis of FMNN}

This section will review the essential concepts of FMNN, and discuss its stability issue. The proposed CFMN will be introduced in Section 3.

The fuzzy min-max clustering neural network $(\mathrm{FMNN})^{12}$ is constructed by using hyperbox fuzzy sets. A hyperbox is completely defined by its $\mathrm{min}$ point and max point. The combination of the minmax points and the hyperbox membership function defines a fuzzy set (cluster). Once the FMNN is trained, it is operated by presenting a pattern and computing the membership value in each of the existing fuzzy sets. Although the learning portion of the algorithm is not neural, the recall operation fits immediately into a neural network framework.

\subsection{Fuzzy Hyperbox Membership Function}

Let the $j$ th hyperbox fuzzy set $B_{j}$, be defined by the ordered set

$$
B_{j}=\left\{A_{h}, V_{j}, W_{j}, b_{j}\left(A_{h}\right)\right\}
$$

for all $h=1,2, \ldots, m$, where $A_{h}=\left(a_{h 1}, a_{h 2}, \cdots, a_{h n}\right) \in$ $\mathbb{I}^{n}$ is the $h$ th pattern in the data set, $V_{j}=$ $\left(v_{j 1}, v_{j 2}, \cdots, v_{j n}\right)$ is the min point for the $j$ th hyperbox, $W_{j}=\left(w_{j 1}, w_{j 2}, \cdots, w_{j n}\right)$ is the max point for the $j$ th hyperbox. An illustration of the hyperbox in $\mathbb{R}^{3}$ is shown in Fig. 1 .

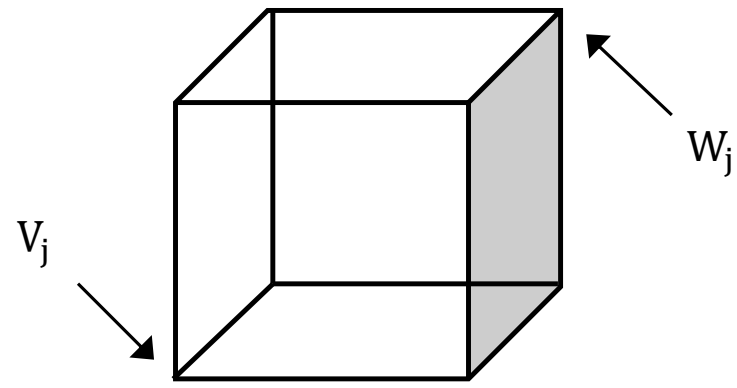

Figure 1: The min-max hyperbox $B_{j}=\left\{V_{j}, W_{j}\right\}$ in $\mathbb{R}^{3}$.

The membership function is defined as

$$
b_{j}\left(A_{h}\right)=\frac{1}{n} \sum_{i=1}^{n}\left[1-f\left(a_{h i}-\omega_{j i}, \gamma\right)-f\left(v_{j i}-a_{h i}, \gamma\right)\right]
$$

where $a_{h i}, \omega_{j i}, v_{j i}$ are the $i$ th element respectively of the vectors $A_{h}, V_{j}, W_{j}$ and $f()$ is the two-parameter ramp threshold function

$$
f(x, \gamma)= \begin{cases}1, & \text { if } x \gamma>1 \\ x \gamma, & \text { if } 0 \leqslant x \gamma \leqslant 1 \\ 0, & \text { if } x \gamma<0\end{cases}
$$

where $\gamma$ is the sensitivity parameter that regulates how fast the membership values decrease as the distance between $A_{h}$ and $B_{j}$ increases. Fig. 2 shows the change of $b_{j}\left(A_{h}\right)$ of one dimension when $\gamma$ changes.

The membership function in FMNN measures the degree to which the $h$ th input pattern $A_{h}$ falls within the hyperbox $B_{j}$ formed by the $\min$ point $V_{j}$ and max point $W_{j}$. On a dimension-by-dimension basis, this can be considered a measurement of how far each component is greater than the max point value or less than the min point value ${ }^{12}$. As $A_{h}$ approaches the hyperbox, $b_{j}\left(A_{h}\right)$ approaches 1 . When the point is contained within the hyperbox, $b_{j}\left(A_{h}\right)$ is equal to 1 . 




Figure 2: Membership function $b_{j}\left(A_{h}\right)$ of FMNN.

\subsection{Recasting FMNN in the Framework of ART}

As a competitive learning based clustering method, FMNN has much similarity with adaptive resonance theory $(\mathrm{ART})^{14}$. ART is a family of neural networks that develop stable recognition categories (clusters) by self-organization in response to arbitrary sequences of input patterns ${ }^{15}$. At the training stage, the stored prototype of a category (cluster) is adapted when an input pattern is sufficiently similar to the prototype. An input pattern is said to be novel to the neural network if it deviates too much from all existing prototypes, then the ART adaptively and autonomously creates a new category with the input pattern as the prototype ${ }^{16}$. An ART system relies on novelty to distinguish between familiar and unfamiliar events (patterns), as well as between expected and unexpected events ${ }^{17}$. FMNN follows the same learning paradigm except that the prototypes are represented by min-max points.

In fact, FMNN grows out of the fuzzification of the ART-1 neural network ${ }^{17}$. Here, for ease of understanding and analysis, we summarize FMNN as a three-layer network in the framework of ART ${ }^{171815}$.



Figure 3: Architecture of FMNN.

The architecture is shown in Fig. 3. The input layer $F 0$ receives and stores the input pattern. The comparison layer $F 1$ stores the normalized input pattern also called the short-term memory. The recognition layer $F 2$ stores the prototypes (min points and max points) of recognition categories (clusters) as the long-term memory. Learning of the FMNN consists of creating and adjusting hyperboxes in the $F 2$ layer, trying to include all patterns in hyperboxes, and is summarized as follows.

\section{Parameters}

The FMNN is determined by the hyperbox size constraint parameter $\theta$, and the parameter $\gamma$ controlling the fuzziness of the membership function which is usually set to 4 .

2. Network Initialization

The $F 2$ layer is initialized as the null set.

3. Input Normalization

The input pattern in the $F 0$ layer is normalized from $\mathbb{R}^{n}$ to $\mathbb{I}^{n}$, and is fed into the $F 1$ layer.

\section{Category Choice}

Given a pattern $A_{h}$ in the $F 1$ layer, the hyperbox $B_{j}$ in the $F 2$ layer that provides the highest degree of membership and allows expansion is identified. The expansion criterion of 
FMNN is

$$
\sum_{i=1}^{n}\left(\max \left(\omega_{j i}, a_{h i}\right)-\min \left(v_{j i}, a_{h i}\right)\right) \leqslant n \theta .
$$

It defines the constraint regulating the average size of the hyperbox. The parameter $\theta$ $(0<\theta<1)$ is a user-defined value directly related to the number of hyperboxes generated in the $F 2$ layer and significantly affects the effectiveness of the training algorithm.

5. Resonance or Reset

Mismatch reset happens when one of the following cases is met:

- Case 1: the first pattern is presented.

- Case 2: all existing $B_{j}$ are exhausted without any expansions.

Then a new category (cluster) $B_{j}$ is created by copying $A_{h}$ as its min point and max point, i.e.,

$$
V_{j}=A_{h} \text { and } W_{j}=A_{h} .
$$

Otherwise the network is said to reach resonance at hyperbox (category) $B_{j}$ found in step 4 ), and the learning begins.

\section{Learning}

The hyperbox $B_{j}$ is updated according to

$$
\begin{gathered}
v_{j i}^{\text {new }}=\min \left(v_{j i}^{\text {old }}, a_{h i}\right), \quad \forall i=1,2, \ldots, n \\
w_{j i}^{\text {new }}=\max \left(w_{j i}^{\text {old }}, a_{h i}\right), \quad \forall i=1,2, \cdots, n .
\end{gathered}
$$

\section{Hyperbox Overlap Test}

This procedure is performed immediately after the previous expansion step. Suppose that $B_{j}$ is expanded, then each dimension of $B_{j}$ is compared with the remaining $B_{k}$. The expansion creates an overlap between $B_{j}$ and $B_{k}$ if there is some kind of overlap for each of the $n$ dimensions.

- Case 1: $v_{j i}<v_{k i}<\omega_{j i}<\omega_{k i}$.

- Case 2: $v_{k i}<v_{j i}<\omega_{k i}<\omega_{j i}$.

- Case 3: $v_{j i}<v_{k i} \leqslant \omega_{k i}<\omega_{j i}$.
- Case 4: $v_{k i}<v_{j i} \leqslant \omega_{j i}<\omega_{k i}$.

Note that the above four cases summarized in Ref. 12 are not complete, and there are four neglected boundary cases which may lead to failure of overlap test, so we restate here with corrections:

- Case 1: $v_{j i}<v_{k i}<\omega_{j i}<\omega_{k i}$.

- Case 2: $v_{k i}<v_{j i}<\omega_{k i}<\omega_{j i}$.

- Case 3: $v_{j i} \leqslant v_{k i} \leqslant \omega_{k i} \leqslant \omega_{j i}$.

- Case 4: $v_{k i} \leqslant v_{j i} \leqslant \omega_{j i} \leqslant \omega_{k i}$.

Each of these cases is illustrated in Fig. 4. The added boundary cases are illustrated by the bottom portions of Fig. 4c and Fig. 4d.

8. Hyperbox Contraction

The overlap between hyperbox $B_{j}$ and $B_{k}$ is eliminated on a dimension-by-dimension basis. Using the four cases described previously, the overlapping hyperboxes are contracted as follows to eliminate overlap in every dimension so that the resulting clusters are more compact.

- Case 1: If $v_{j i}<v_{k i}<\omega_{j i}<\omega_{k i}$,

$$
v_{k i}^{\text {new }}=\omega_{j i}^{\text {new }}=\frac{v_{k i}^{\text {old }}+\omega_{j i}^{\text {old }}}{2} .
$$

- Case 2: If $v_{k i}<v_{j i}<\omega_{k i}<\omega_{j i}$,

$$
v_{j i}^{\text {new }}=\omega_{k i}^{\text {new }}=\frac{v_{j i}^{\text {old }}+\omega_{k i}^{\text {old }}}{2} .
$$

- Case 3: If $v_{j i} \leqslant v_{k i} \leqslant \omega_{k i} \leqslant \omega_{j i}$, then the contraction is performed on the smaller of the two overlaps. If $\omega_{k i}-v_{j i}<\omega_{j i}-v_{k i}$ then contract using the assignment

$$
v_{j i}^{\text {new }}=\omega_{k i}^{\text {old }},
$$

otherwise use the assignment

$$
\omega_{j i}^{\text {new }}=v_{k i}^{\text {old }} .
$$

- Case 4: If $v_{k i} \leqslant v_{j i} \leqslant \omega_{j i} \leqslant \omega_{k i}$, then, by symmetry, the same assignments under the same conditions in case 3 are applied here. 




(a) Case 1: Overlap $=w_{j i}-v_{k i}$

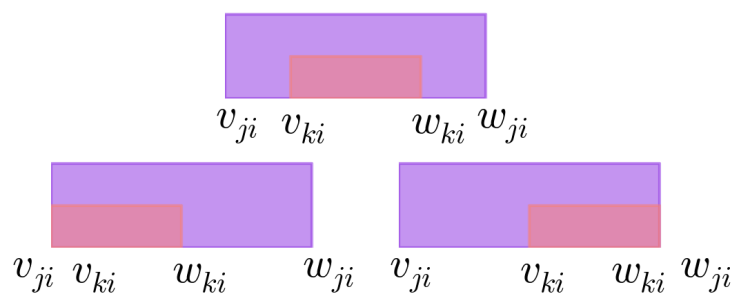

(c) Case 3: Overlap $=\min \left(w_{j i}-v_{k i}, w_{k i}-v_{j i}\right)$



(b) Case 2: Overlap $=w_{k i}-v_{j i}$

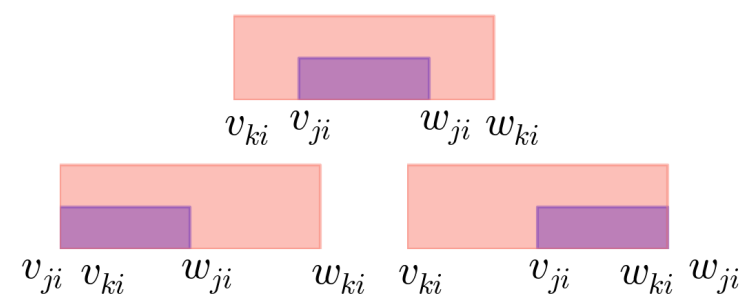

(d) Case 4: Overlap $=\min \left(w_{j i}-v_{k i}, w_{k i}-v_{j i}\right)$

Figure 4: The four cases that occur during overlap testing. Each graph shows the relative values of two hyperboxes along one dimension. Part of this figure is adapted from FMNN.

\section{Cluster Stability Test}

Steps 3)-8) are repeated for each pattern in the data set until cluster stability is achieved. Cluster stability is defined as all hyperbox min and max points not changing during successive presentations of the dataset in the same order.

Please note that steps 4)-6) together corresponds to the hyperbox expansion procedure of the original FMNN.

\subsection{The Stability Issue of FMNN}

In FMNN, the inappropriate setting of $\theta$ and the randomness present in the dataset may slow down the learning algorithm and even prevent it from convergence. This is because that the expansion for patterns with low membership degree makes the clusters easy to grow up to full size, resulting in overlaps between clusters. The situation would become much more worse when many points are at the cluster boundary.

After exploring the algorithm for different parameters and datasets, we find an interesting phe- nomenon that the prototypes generated will reappear after every $p(p \geqslant 1)$ presentations of the data set in step 9) of the training process, i.e., the overlap contraction process has some kind of periodicity. At the beginning of the next presentation of the dataset (represented by $D$ ), the cluster learning algorithm is based on the result of the last presentation. Hence the clusters eventually resulted are based on the dataset $[D, D, \cdots, D]$ of length $p$, not $D$ if $p>1$, and thus the clusters are not well-defined.

The above stability issue can be explained by the well-known stability-plasticity dilemma ${ }^{17}$ in the ART literature. Simply speaking, a system must have plasticity to learn about significant new events, and it must also remain stable in response to irrelevant or often repeated events. In Ref. 19, two types of stability condition named as stable 1 and stable $_{2}$ are defined for incremental clustering algorithms:

1. stable $_{1}$ : No prototype vector can "cycle", or take on a value that it had at a previous time.

2. stable $_{2}$ : Only a finite number of clusters are formed with infinite presentation of the data set. 
FMNN fails to meet stable $_{1}$ because FMNN tries to include all patterns in hyperboxes which makes the neural network too plastic.

Since we will employ the clustering neural network to generate rules for fuzzy influence system, the clustering algorithm should overcome the stability-plasticity dilemma and converge quickly. More importantly, noisy data from real world would lead to serious stability issue.

Previous discussion suggests that FMNN should be modified, so that the neural network can overcome the stability-plasticity dilemma and still performs well under noisy environment. Therefore, we introduce a new FMNN named contribution-factor based fuzzy min-max neural network (CFMN), which achieves more stability by restricting the plasticity and is more robust.

\section{Proposed CFMN Clustering Algorithm}

A common feature of incremental clustering algorithms is that the resulting clusters would be different if the presentation order of the data set changes. This feature will be utilized in CFMN to capture the high-density region of a cluster when the cluster is corrupted by noise. Moreover, the algorithm's plasticity will be restricted to overcome the stabilityplasticity dilemma.

\subsection{Overview of CFMN}

The main characteristics of CFMN are summarized as follows:

- The parameters are intuitive to choose.

- As clustering is exploratory in nature, it's often the case that the algorithm estimates the wrong number of clusters. CFMN can ensure that the estimated cluster centers are around the real ones when the parameters are set over an appropriate range, i.e., the estimated clusters are not affected by the clusters which the algorithm fails to discover.

- CFMN exploits the order-dependent feature of incremental clustering to make the algorithm orderindependent, i.e., the hyperboxes are designed to enclose the high-density regions of clusters, and this holds even when the data set is shuffled.

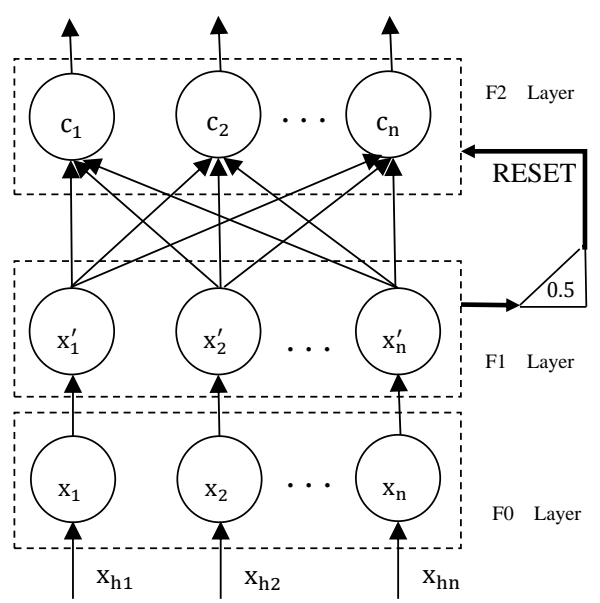

(a)

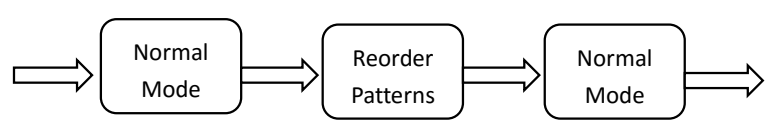

(b)

Figure 5: Architecture of CFMN. (a) Normal mode. (b) Relearning mode.

The architecture of CFMN is shown in Fig. 5. Compared with FMNN, CFMN makes the mismatch reset condition explicit and has two operation modes, i.e., normal mode and relearning mode.

CFMN works in normal mode if data are generated and provided in sequence. This mode is based on the observation that data sets generated in real world represent some behavior of the underlying system and, patterns are often not equally affected by noise. Furthermore, normal (or typical) patterns are more likely to be generated first and are also more likely to form high-density regions. In contrast, patterns affected too much by noise (noisy patterns) often form the low-density region of a cluster. More specifically, each cluster is assumed to consist of one high-density region and one or several low-density regions, and CFMN aims to enclose 
typical patterns in hyperboxes without influence of low-density regions.

The relearning mode is chosen if the data set loses the order information. As the patterns presented first to the algorithm are closely connected to the resulting clusters, CFMN introduces the relearning mechanism to eliminate the impact of presentation order.

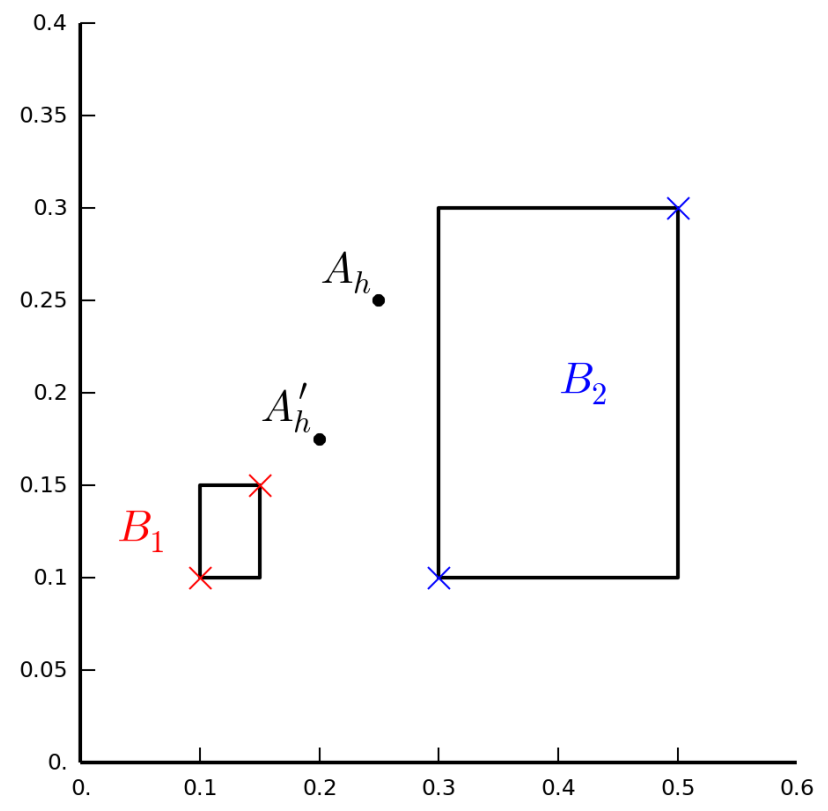

Figure 6: Expansion of patterns. In CFMN, $A_{h}$ is neglected and only $A_{h}^{\prime}$ can be expanded to $\mathrm{B}_{1}$.

The development of CFMN has considered the following requirements.

1. The hyperbox should only expand typical patterns.

Hyperbox expansion is an important step in the learning algorithm and it is closely related to coping with noisy patterns. Suppose that the high-density region of a cluster is first presented to the algorithm so that a hyperbox has been created for that region. Then it's reasonable to consider patterns with low membership degree to existing clusters as noisy data. An example is shown in Fig. 6. Pattern $A_{h}$ is presented to the network before $A_{h}^{\prime}$. We can see that $A_{h}$ is closer to the hyperbox $B_{2}$ than $B_{1}$. However, $B_{2}$ can't meet the expansion criterion. Furthermore, $A_{h}$ contains too much noise to be expanded for $B_{1}$. In contrast, $A_{h}^{\prime}$ can be seen as a typical pattern for $B_{1}$ and can be expanded. $A_{h}$ is neglected and doesn't contribute to the expansion of any hyperboxes in CFMN, whereas in FMNN, $A_{h}$ can be included into $B_{1}$, which makes the hyperbox grow to full size quickly and the expansion sensitive to noisy patterns.

2. The membership function should reflect the noise degree of a pattern.

The membership function of FMNN shown in Fig. 2 is calculated based on the pattern's distance to the cluster boundary, i.e., $b_{j}\left(A_{h}\right)$ is close to 1 if $A_{h}$ is close to the min point or max point. Then a pattern far from the cluster center can have high membership to the cluster. The authors of DCFMNN ${ }^{20}$ pointed out that the noisy data near the boundary should also be taken care of. The boundary-based membership function is widely used in the fuzzy min-max neural network literature. However, the membership degree changes dramatically with the boundary as the cluster expands. This fact suggests that it's difficult to use the boundary-based membership function as an indicator to detect noisy data. For example, the noise level is first estimated from data in DCFMNN ${ }^{20}$. Then, $b_{j}\left(A_{h}\right)$ of the pattern $A_{h}$ near the boundary is lower if the noise level is high.

As stated previously in 1), the cluster center has been supposed to lie in the high-density region. The pattern far from the cluster center often lie in the low-density region, so the data near the cluster boundary should also have low membership degree. This is achieved by implementing an exponential membership function, which makes it easier for CFMN to copy with noisy data and the detection of outliers.

3. The resulting clusters should be wellseparated. 


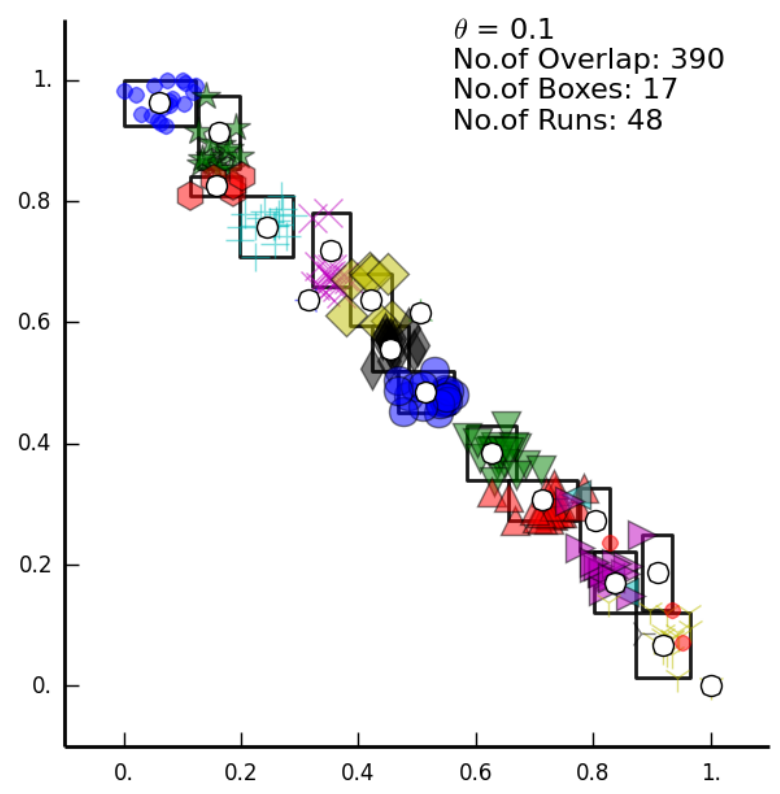

(a)

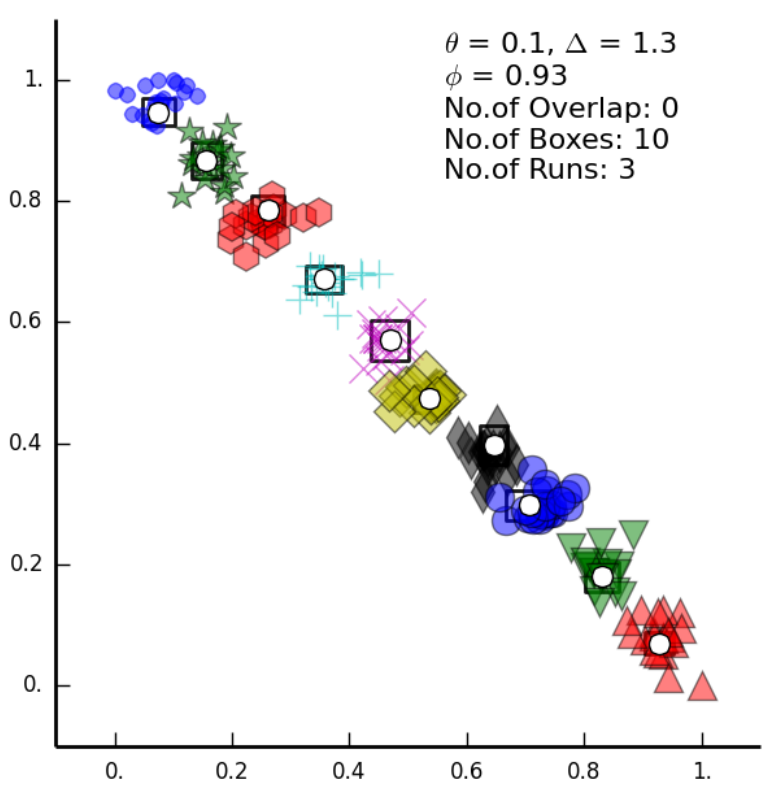

(b)

Figure 7: Comparison of FMNN and CFMN on a data set with 10 clusters provided in sequence. The center of each cluster is presented first. (a) Result of FMNN. (b) Result of CFMN.

Choosing parameters in FMNN lacks intuition and the generated hyperboxes are not always well-separated. This can be seen in Fig. 7a. CFMN introduces a parameter to control the inter-cluster distance. The clusters in Fig. 7b is more well-separated than in Fig. 7a. Fig. 7 also shows that the overlaps can be avoided in CFMN.

\subsection{Basic Definitions}

In the following, we will describe the concepts of novelty, virtual boundary, and typical pattern respectively, which are essential in the development of CFMN.

Let $A_{h 1}$ and $A_{h 2}$ denote the input patterns, $c_{i}$ and $c_{j}$ denote the cluster centers of clusters $B_{i}$ and $B_{j}$, and $V_{j}$ and $W_{j}$ denote the min-max points of cluster $B_{j}$. The center $c_{j}$ is defined as $\left(V_{j}+W_{j}\right) / 2 . \theta$, $d_{0}, d_{1}$, and $d_{2}$ are parameters controlling the behavior of the network. $\theta$ specifies the maximum cluster size for each dimension in an average sense (note that parameter $\theta$ of FMNN specifies the maximum hyperbox size).

The distance between $A_{h 1}$ and $A_{h 2}$ is defined as

$$
d\left(A_{h 1}, A_{h 2}\right)=\left(\left\|A_{h 1}-A_{h 2}\right\|^{2}\right)^{1 / 2} .
$$

Pattern $A_{h}$ is novel to the neural network if

$$
\min _{j} d\left(A_{h}, c_{j}\right) \geqslant d_{0} .
$$

Then a new hyperbox (cluster) will be created for $A_{h}$, and $d_{0}$ can be interpreted as the minimum distance between neighboring cluster centers, i.e.,

$$
\min _{i, j} d\left(c_{i}, c_{j}\right) \approx d_{0} .
$$

The cluster boundary in FMNN is formed by the min-max points, whereas in CFMN, the hyperbox formed by the min-max points are used to enclose only a portion of the cluster. So the virtual boundary of a cluster $B_{j}$ is defined as the points that satisfy

$$
d\left(A_{h}, c_{j}\right)=d_{1}
$$

where $d_{1}$ is defined as $\frac{1}{2} d_{0}$. 
$A_{h}$ is a typical (normal) pattern to $B_{j}$ if

$$
d\left(A_{h}, c_{j}\right) \leqslant d_{2}
$$

where $d_{2} \leqslant d_{1}$, i.e., a typical pattern should lie in the virtual boundary of a cluster.

Before proceeding, we shall introduce a parameter $\Delta$ which satisfies

$$
\begin{aligned}
& d_{0}=\Delta \cdot \theta \\
& d_{1}=\frac{1}{2} \Delta \cdot \theta .
\end{aligned}
$$

Next, parameters $d_{0}, d_{1}$, and $d_{2}$ will be converted so that they are related to the membership degree of a pattern and more intuitive for the user to choose.

As stated previously, the membership function should decrease with the distance between pattern $A_{h}$ and the centroid of hyperbox $B_{j}$ in order to make it an indicator to tell good data from noisy data or outliers. To meet the required criterion, a new membership function is defined as

$$
\begin{aligned}
b_{j}\left(A_{h}\right) & =\exp \left\{-\frac{d^{2}\left(A_{h}, c_{j}\right)}{\gamma^{2}}\right\} \\
& =\exp \left\{-\frac{\left\|A_{h}-c_{j}\right\|^{2}}{\gamma^{2}}\right\}
\end{aligned}
$$

where $\gamma$ is a value that satisfies

$$
\exp \left\{-\frac{d_{0}^{2}}{\gamma^{2}}\right\}=\exp \left\{-\frac{(\Delta \cdot \theta)^{2}}{\gamma^{2}}\right\}=0.5
$$

Note that $\theta$ and $\Delta$ are user-specified parameters.

The membership threshold 0.5 has similar functions as the vigilance value in ART below which a new category (cluster) is created, i.e., $A_{h}$ is novel to the network if $\min _{j} b_{j}\left(A_{h}\right) \leqslant 0.5$. As we know, the creation of new hyperboxes is closely related to the detection of outliers, i.e., we shall create a new hyperbox $B_{j}$ at the distance of $\Delta \theta . B_{j}$ is either a cluster of true patterns or a cluster of outliers, and the difference is that the density is lower in the latter case. Here we explicitly relate the membership function and the creation of new hyperboxes to $\theta$, which makes the selection of $\theta$ easier for the user.

For the pattern $A_{h}$ at the virtual boundary of hyperbox $B_{j}$, we can get its membership degree from
(14), (16), and (18)

$$
\begin{aligned}
b_{j}\left(A_{h}\right) & =\exp \left\{-\frac{d_{1}^{2}}{\gamma^{2}}\right\} \\
& =\exp \left\{-\frac{(0.5 \Delta \cdot \theta)^{2}}{\gamma^{2}}\right\} \\
& \approx 0.84 .
\end{aligned}
$$

From (15) and (19), a typical pattern $A_{h}$ has membership degree

$$
b_{j}\left(A_{h}\right) \geqslant \phi=\exp \left\{-\frac{d_{2}^{2}}{\gamma^{2}}\right\} \geqslant 0.84 .
$$

In CFMN, only patterns with high membership degree (i.e., typical patterns) are allowed to contribute to the adjustment of a hyperbox. To emphasize this characteristic, a typical pattern of cluster $B_{j}$ is also called a contribution-factor $(C F)$ to $B_{j}$. In fact, $C F M N$ introduces $C F$ to limit the network's plasticity, i.e., only the $C F$ (with strong influence to some neuron) can be turned into long-term memory, thus overcoming the stability-plasticity dilemma.

\subsection{CFMN in Normal Mode}

The two learning modes will be introduced separately. The learning process of normal mode is similar with that of FMNN. We will only describe its main part.

\section{Parameters}

CFMN has three parameters. $\theta$ specifies the maximum cluster size for each dimension in an average sense, $\Delta$ specifies the ratio of the minimum inter-cluster distance relative to $\theta$, and $\phi$ specifies the membership threshold above which a pattern can be a $C F$ of a cluster $(\phi \in(0.84,1)$ because a $C F$ must lie in the virtual boundary of a cluster).

2. Network Initialization

The $F 2$ layer is initialized as the null set.

\section{Input Normalization}

The input pattern in the $F 0$ layer is normalized to $\mathbb{I}^{n}$, then it is fed into the $F 1$ layer. This step is optional since the membership function is of Gaussian type. 


\section{Category Choice}

Given a pattern $A_{h}$ in the $F 1$ layer, the hyperbox $B_{j}$ in the $F 2$ layer that provides the highest degree of membership and allows expansion is identified. The expansion criterion is

$$
\left\{\begin{array}{l}
\sum_{i=1}^{n}\left(\max \left(\omega_{j i}, a_{h i}\right)-\min \left(v_{j i}, a_{h i}\right)\right) \leqslant n \theta \\
b_{j}\left(A_{h}\right) \geqslant \phi .
\end{array}\right.
$$

It implies that the cluster $B_{j}$ shouldn't expand for $A_{h}$ if $B_{j}$ becomes too full after expansion, or if $A_{h}$ is not a $C F$ (typical pattern) of $B_{j}$.

5. Resonance or Reset

Mismatch reset happens when one of the following cases is met:

- Case 1: the first pattern is presented.

- Case 2: $\max _{j} b_{j}\left(A_{h}\right) \leqslant 0.5$., i.e., novelty is detected.

Then a new category (cluster) $B_{j}$ is created by copying $A_{h}$ as its min point and max point, i.e.,

$$
V_{j}=A_{h}^{l} \text { and } W_{j}=A_{h}^{u} .
$$

Otherwise the network is said to reach resonance at hyperbox (category) $B_{j}$ found in step 4 ), and the learning begins.

\section{Learning}

\section{Hyperbox Overlap Test}

8. Hyperbox Contraction Steps 6)-8) are same with that of FMNN.

\section{Cluster Stability Test}

Steps 3)-8) are repeated for each pattern of the data set in the same order until cluster stability is achieved. Note that the stability issue encountered in FMNN may occur here when the network has large plasticity, i.e., $\phi$ is set around 0.84, which implies that patterns along the virtual boundary are allowed to be expanded. Increasing the value of $\phi$ can solve this issue and can also significantly speed up the learning process.

\subsection{CFMN in Relearning Mode}

Clustering algorithms such as the K-Means and Expectation Maximization, require to specify the number of clusters before the clustering process. In contract, FMNN and CFMN require to specify the cluster size of individual dimension. Just as K-Means and Expectation Maximization that are sensitive to the initialization of cluster centers, the order of input patterns during training affects the nodes (clusters) in the $F 2$ layer of CFMN or FMNN, which is a common feature for incremental learning algorithms. The common method to solve this problem is to present training patterns in random order, where voting strategy is used to compute the final performance. Genetic algorithm and Particle Swarm Optimization have also been used to select the presentation order of training patterns ${ }^{2122}$.

As stated previously, the presentation order is utilized in normal mode of CFMN to capture the high-density region of a cluster when the cluster is corrupted by noise and the data set is provided in sequence. However, the high-density region can't be captured perfectly when the data set loses the order information, which is often the case.

The introduction of $C F$ can limit the plasticity of the network, i.e., a hyperbox can only expand near patterns, and its center will not move dramatically since it's created if the user specifies a large $\phi$. The relearning mode relies on this fact to capture the high-density region. The architecture of this mode is shown in Fig. 5b. The patterns are first clustered in CFMN normal mode, then patterns in each cluster are sorted by density before applying CFMN normal mode again. In the second applying of CFMN normal mode, the high-density region of each cluster is presented first and enclosed by the hyperbox. Density estimation is performed using the kernel density estimation $(\mathrm{KDE})^{23}$

$$
p(x)=\frac{1}{N} \sum_{n=1}^{N} \frac{1}{\left(2 \pi h^{2}\right)^{1 / 2}} \exp \left\{-\frac{\left\|x-x_{n}\right\|^{2}}{2 h^{2}}\right\} .
$$

where $h$ denotes the bandwidth ( $h$ is set to $\theta / 20$ in this study).

The relearning mechanism can ensure that the resulting clusters are not affected too much by the 
noisy patterns. For illustration, this mode is run on the popular ruspini ${ }^{24}$ data set. The data set is shuffled before clustering. The result is shown in Fig. 8 . The high-density region of each cluster is perfectly captured by the hyperbox and the cluster represented by stars in the figure can be seen as an outlier cluster.



Figure 8: Result of CFMN on the ruspini data set. The data set is shuffled before clustering.

\section{CFMN Fuzzy Inference System}

The clustering algorithm has been introduced in Section 2 and Section 3. Now we will concentrate on the construction of fuzzy models.

A fuzzy system consists of a bunch of fuzzy if-then rules. Generally, fuzzy system identification consists of structure identification and parameter learning. Structure identification is used to determine the fuzzy membership functions and the appropriate number of rules. Parameter learning estimates the coefficients of each rule.

In the proposed CFMN fuzzy inference system (CFMN-FIS), the clusters generated by CFMN are used as fuzzy rules. That is, the premise parameters (membership functions of input variables) are specified by the clusters and the input parameters of CFMN. Note that CFMN works in normal mode when the data set is provided in sequence. Otherwise CFMN works in relearning mode. The resulting fuzzy system is of the following Takagi-SugenoKang (TSK) form:

$$
\begin{aligned}
& \text { Rule } j: \operatorname{IF} x_{1} \text { is } A_{j 1} \text { AND } \cdots \text { AND } x_{n} \text { is } A_{j n} \\
& \quad \operatorname{THEN} y^{j}=b_{0}^{j}+b_{1}^{j} x_{1}+\cdots+b_{n}^{j} x_{n} \quad(1 \leqslant j \leqslant c) \\
& =(1, x) \cdot\left(b_{0}^{j}, \cdots, b_{n}^{j}\right)^{T}
\end{aligned}
$$

where $x=\left(x_{1}, \cdots, x_{n}\right)$ is the input pattern, $A_{j}$ is the $j$ th hyperbox (cluster), and $\mathrm{b}_{\mathrm{i}}^{\mathrm{j}}(0 \leqslant i \leqslant n)$ are consequent parameters.

For the $j$ th Rule that is represented by cluster center $c_{j}$ (middle point of the min and max points $V_{j}, W_{j}$ ), fuzzy set $A_{j i}$ is given by

$$
A_{j i}\left(x_{i}\right)=\exp \left\{-\left(\frac{x_{i}-\left(w_{j i}+v_{j i}\right) / 2}{\gamma}\right)^{2}\right\}
$$

where $\gamma$ is defined in (18).

The fire strength $\mu_{j}(x)$ of Rule $j$ is calculated by using multiplication as the AND operator

$$
\mu_{j}(x)=\prod_{i=1}^{n} A_{j i}\left(x_{i}\right)
$$

The inferred output of the model is calculated as

$$
\begin{aligned}
\widehat{y} & =\frac{\sum_{j=1}^{c} \mu_{j}(x) y^{j}}{\sum_{j=1}^{c} \mu_{j}(x)} \\
& =\left(\rho^{1}, \rho^{1} x, \cdots, \rho^{c}, \rho^{c} x\right) \cdot\left(b_{0}^{1}, \cdots, b_{n}^{1}, \cdots, b_{0}^{c}, \cdots, b_{n}^{c}\right)^{T}
\end{aligned}
$$

where $\rho^{j}=\mu_{j}(x) / \sum_{j=1}^{c} \mu_{j}(x)$. The consequent parameters are then estimated by the recursive least squares (RLS) algorithm.

\section{Experimental Results}

Demonstration of CFMN and CFMN-FIS will now be given on a variety of data sets. The first two examples relate to clustering characteristics of CFMN and the remaining three relate to CFMN-FIS. FMNN-FIS is also constructed in the same way as CFMM-FIS for comparison. 


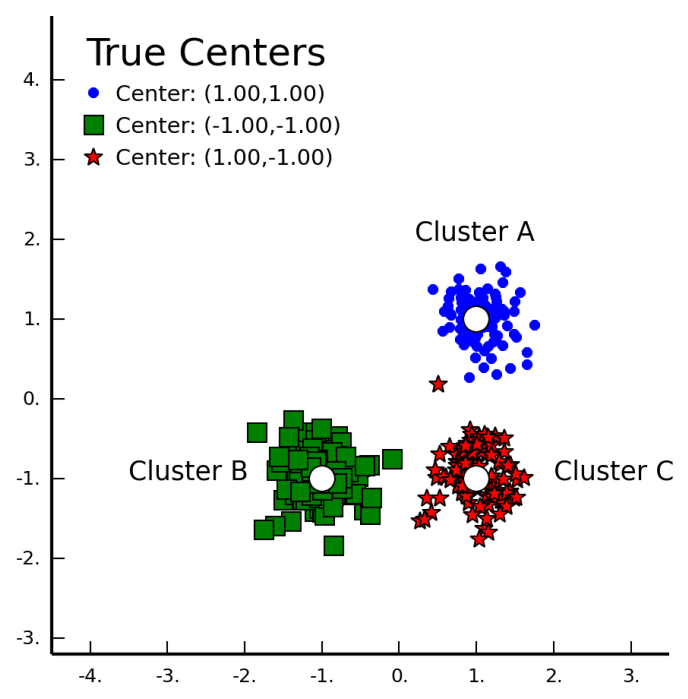

(a)

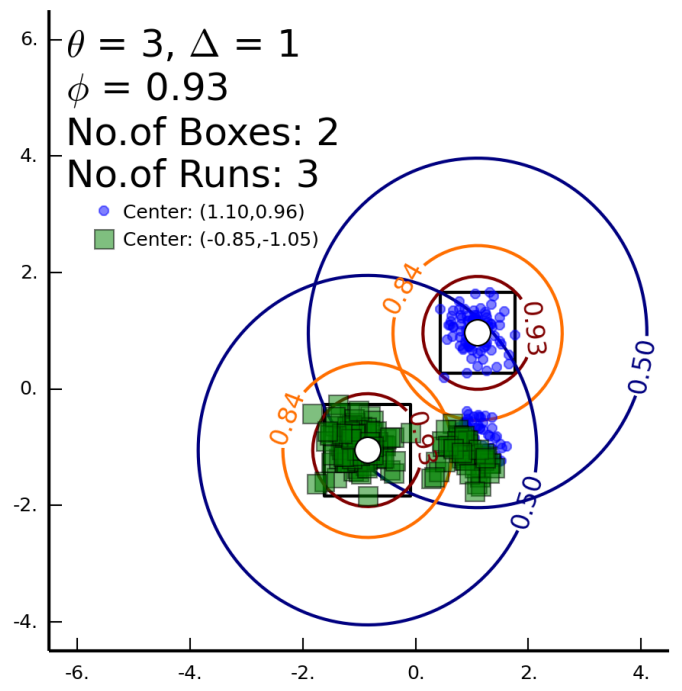

(c)

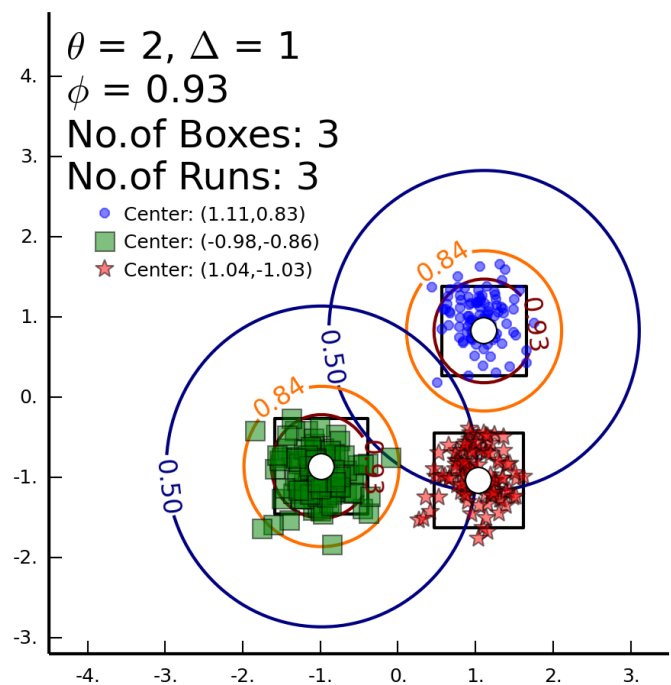

(b)

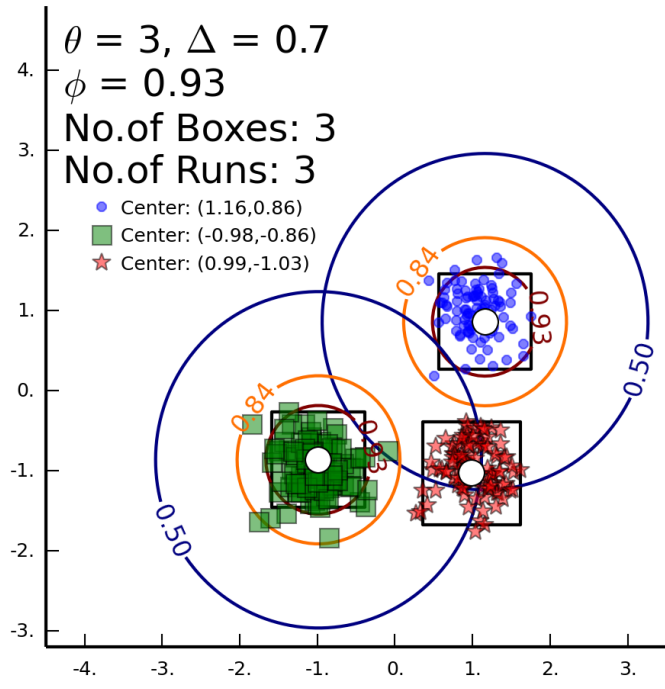

(d)

Figure 9: Clustering result of Example 1. The centers estimated by CFMN are represented by big circles and the hyperboxes are represented by rectangles.

\subsection{Example 1-the Order-dependent Feature of CFMN}

The data used in this example is constructed in order to illustrate the order-dependent feature of CFMN. More specifically, the estimated cluster centers are around the real ones when the parameters are set over an appropriate range, i.e., the estimated clusters are not affected by the clusters which the algorithm fails to discover. We also show that the introduction of $\Delta$ offers the user more control over the clustering process. This data set consists of 100 input patterns for each cluster. It's generated by 3 Gaussian 
blobs which are denoted by Cluster A, B, C with standard deviation 0.3 and with centers $[(1,1),(-1$, $-1),(1,-1)]$ respectively. The true clusters are shown in Fig. 9a. We can see that the hyperbox size is approximately 2 and the inter-cluster distance 2 . So the parameters of CFMN can be chosen as $[\theta=2, \Delta$ $=1, \phi=0.93]$. Please note that the setting of $\phi$ here is arbitrary and the training will be fast if $\phi$ is large because the number of CF will be very small.

To illustrate the benefit of $\Delta$ 's control over the clustering process, the training of the network for two different settings of $\theta$ has been carried out. The clusters are presented to the algorithm in the generation order: [Cluster A, Cluster B, Cluster C]. The parameters and clustering results including the estimated centers are shown in Fig. 9. Contour plots of the membership function with three levels [0.5, $0.84,0.93$ ] are also shown, and for clarity, only two clusters in each subfigure are plotted with contours. Note that 0.5 is a threshold to determine if the pattern is novel to the network and the virtual boundary of each cluster is represented by the membership value with level of 0.84

Fig. 9b shows the results of appropriate setting of parameters. In Fig. 9c, $\theta$ is set to 3 , which is apparently too large and results in only two clusters, but it's interesting to observe that the two estimated cluster centers are still very close to the real ones. The reason why the estimated centers of Cluster A and Cluster B are not affected by Cluster $\mathrm{C}$ is that in CFMN clusters evolve by expansion of CFs. The evolving stops because of the lack of CF caused by gaps between pattern regions. In Fig. 9d, the undesirable effects of large $\theta$ is fortunately avoided when we adjust $\Delta$ to 0.7 . Although the clusters are almost the same in Fig. 9b and Fig. 9d, the clusters are allowed to grow a bit larger in the latter case because of larger $\theta$.

For comparison, the results of FMNN is shown in Fig. 10. In Fig. 10a, points that belongs to Cluster $\mathrm{C}$ are wrongly enclosed by Cluster B. In Fig. 10b, FMNN also estimates 2 clusters when $\theta=3$, however, Cluster $\mathrm{A}$ and Cluster $\mathrm{C}$ are merged and the estimated cluster center is also affected by this merge.

This example shows that the utilization of orderdependent information enables CFMN to discover the high-density regions of clusters more robustly than FMNN. This feature also allows CFMN to generate fuzzy rules in a more reasonable way for a wider range of parameters than FMNN. In other words, the input space of the system can be more easily covered. In order to exploit the orderdependent information, CFMN also improves the noise-resistant capability, as will be seen in the next example.

\subsection{Example 2-the Stability Feature and the Enhanced Error-rejection Characteristic of CFMN}

This example is included to illustrate the stability feature and the enhanced error-rejection characteristic of CFMN. The stability issue of FMNN is addressed by CFMN via restricting the plasticity with parameter $\phi$. More specifically, CFMN introduces the CF strategy to reduce the unnecessary overlaps in FMNN, and also gains more stability over FMNN. We reproduce the dataset of GFMM ${ }^{13}$. The original data set consists of 41 patterns, 40 of which were randomly generated around point $(0.25,0.25)$ within 0.05 range, and the remaining one represented an outlier. We add a small cluster of 20 patterns on the right in order to make sure that the when the algorithm's parameters are chosen to detect outliers, the algorithm should still produce reasonable clusters. Fig. 11 illustrates the advantage of CFMN. The outlier can be detected at very large $\theta$ whereas FMNN suffers the problem at large $\theta$. We can also see that CFMN avoids the unnecessary overlaps in FMNN. Please note that hyperbox overlaps directly reflect the plasticity of the network, and thus can indicate the stability.

\subsection{Example 3-Identification of the Dynamic System}

In this example, CFMN-FIS is used to identify a dynamic system described by Juang and $\operatorname{Lin}^{25}$.

$$
y(t+1)=\frac{y(t)}{1+y^{2}(t)}+u^{3}(t) .
$$

For the training of CFMN-FIS, the inputs are $y(t)$ and $u(t)$, and the desired output is $y(t+1)$. Train- 


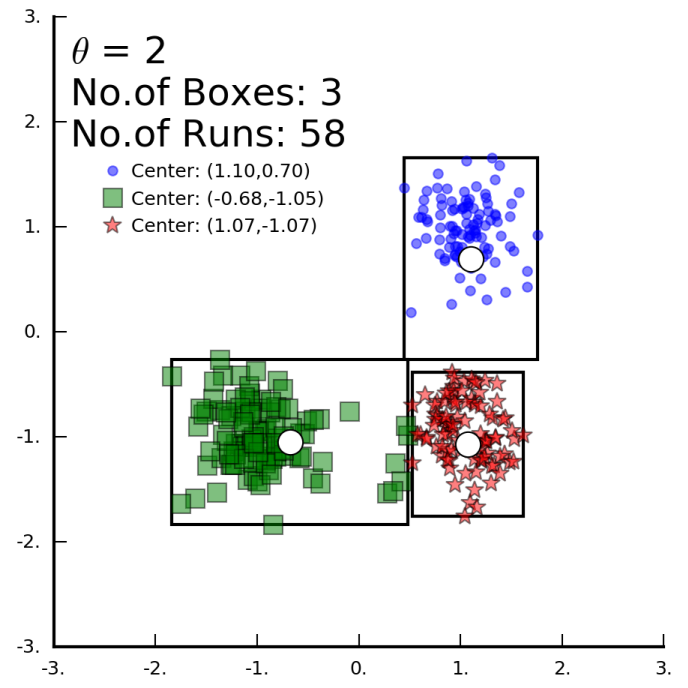

(a)

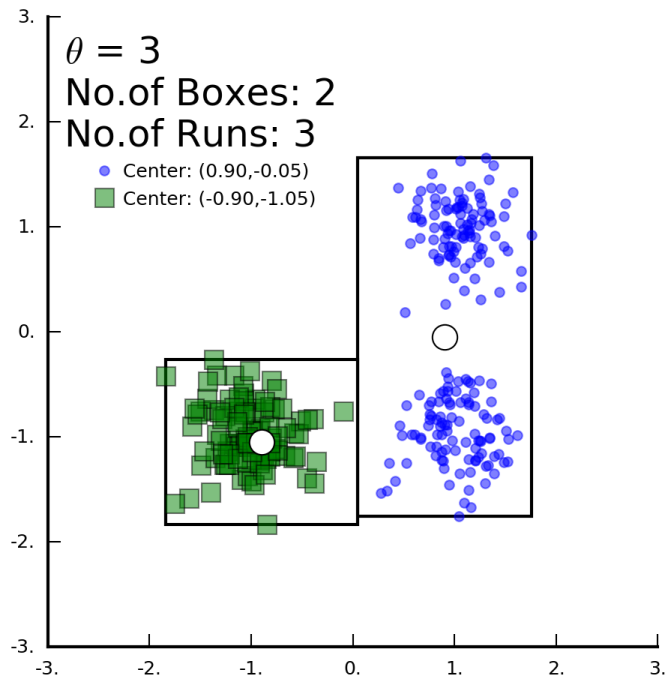

(b)

Figure 10: The clustering result of FMNN on dataset Fig. 9a.

ing patterns are generated with $u(t)=\sin (2 \pi t / 100)$ and $y(0)=0$. This system has been widely used in the literature with different training size and same testing size (200). The popular choice of training size is 200 and 50000. For comparison, CFMNFIS is run in both cases. Fig. 12 shows distributions of the fuzzy sets in the input variables when there are 11 clusters. To test the performance of CFMN-FIS when the number of clusters vary, we choose three sets of parameters, i.e., CFMN-FIS ${ }^{\mathrm{a}}$ : $[\theta=0.25, \phi=0.93, \Delta=1]$, CFMN-FIS ${ }^{\mathrm{b}}:[\theta=0.15$, $\phi=0.97, \Delta=1]$, and CFMN-FIS ${ }^{\mathrm{c}}:[\theta=0.05, \phi$ $=0.963, \Delta=2]$. FMNN-FIS is run with $\theta=0.15$. Table 1 shows the performances of CFMN-FIS and other algorithms. The results of RIT2NFS-WB and SEIT2FNN are adapted from Ref. 26, the results of McIT2FIS-US and eTS are adapted from Ref. 27, the result of SAFIS is adapted from Ref. 28, the result of SONFIN is adapted from Ref. 25, the result of McFIS is adapted from Ref. 29.

From Table 1 we can see that CFMN-FIS can generalize well to a small $K$, and achieves state-ofthe-art results on this dataset. Note that, this dataset is provided in sequence. FMNN-FIS also captures the order-dependent information. However, the performance of FMNN-FIS with training size 200 is not good as RIT2NFS-WB and SEIT2FNN. In contrast, the performance of CFMN-FIS is good in both training cases. This example shows that CFMN-FIS has good performance on datasets provided in sequence because of the utilization of order-dependent information.

\subsection{Example 4-Mackey-Glass Time-Series Prediction}

This example studies the prediction of the following Mackey-Glass chaotic time series:

$$
\frac{d x(t)}{d t}=\frac{0.2 x(t-\tau)}{1+x^{10}(t-\tau)}-0.1 x(t) .
$$

Parameter $\tau$ is set to 17 , and $x(0)=1.2$. We extract 1000 input-output pairs of the following format $[x(t-18), x(t-12), x(t-6), x(t) ; x(t+6)]$ where $t=$ 118 to 1117 . The first 500 samples are used for training and remaining 500 are used for testing. This is the same experimental setting as Ref. 30. CFMNFIS is run for three sets of parameters, i.e., CFMNFIS $^{\mathrm{a}}:[\theta=0.18, \phi=0.93, \Delta=1]$, CFMN-FIS $^{\mathrm{b}}:[\theta=$ $0.14, \phi=0.96, \Delta=1]$, and CFMN-FIS ${ }^{\mathrm{c}}:[\theta=0.12, \phi$ $=0.87, \Delta=1]$. FMNN-FIS is run with $\theta=0.14$. The results are shown in Table 2 . The performance of 


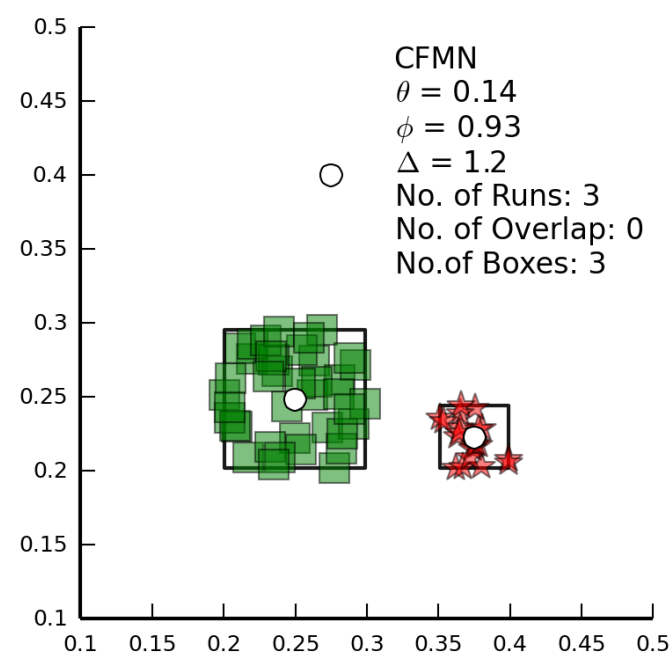

(a)

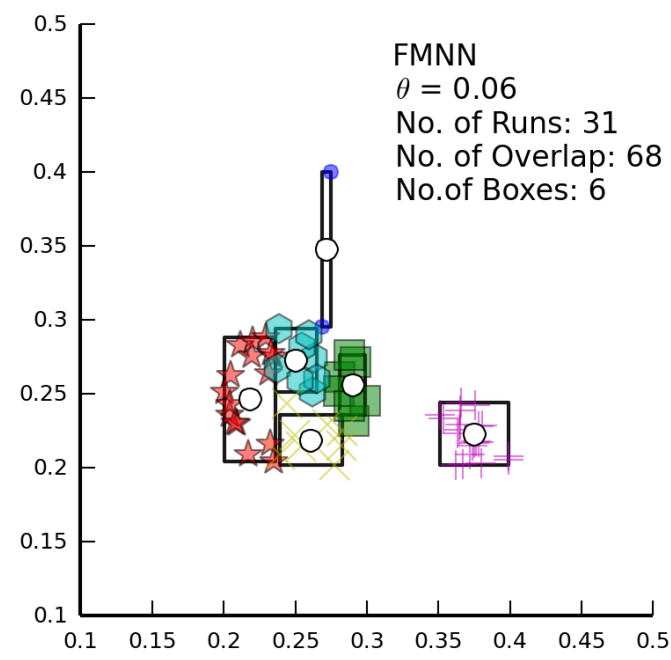

(c)

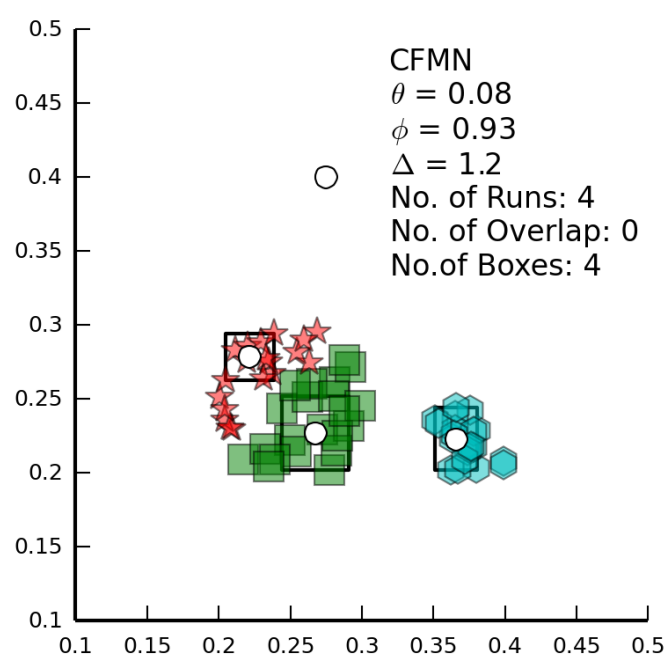

(b)

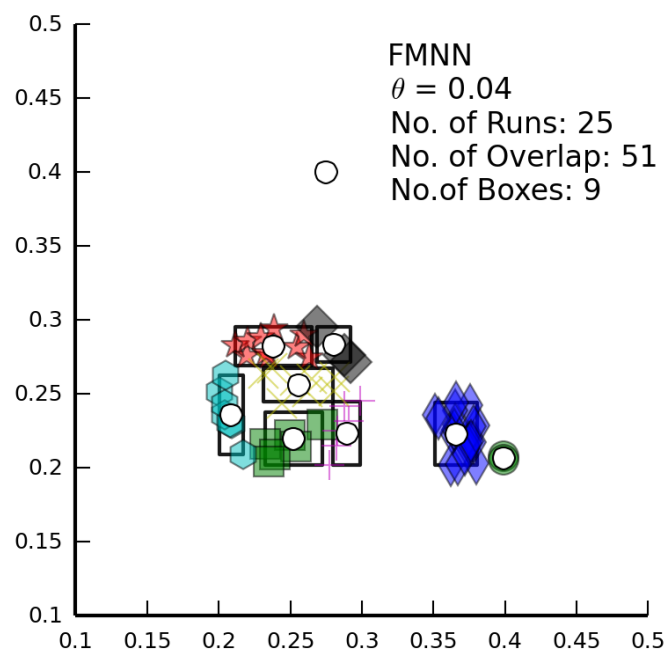

(d)

Figure 11: Clustering in presence of outlier.

CFMN-FIS is benchmarked against the following algorithms: ANFIS ${ }^{30}$, GEFREX $^{31}, \mathrm{RBF}^{32}, \mathrm{RBF}^{33}$, and RBF-AFS ${ }^{34}$. The result of CFMN-FIS is better than FMNN-FIS and other algorithms. This example again shows the advantage of capturing the order-dependent information of data when the data are provided in sequence. 


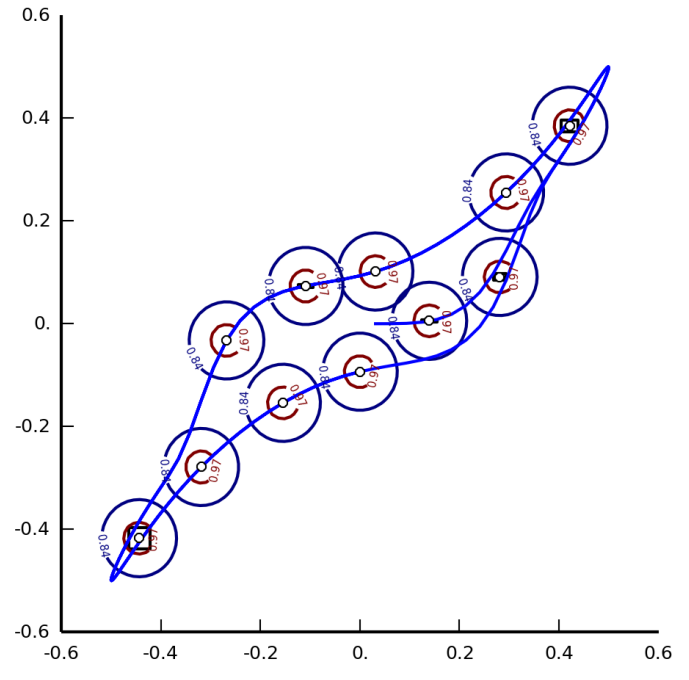

Figure 12: Input space and final assignment of rules corresponding to CFMN-FIS ${ }^{\mathrm{b}}$ of Example 3.

Table 1. Performances of various fuzzy models for the dynamic system of Example 3. $N$ represents the training size, $K$ represents the number of rules.

\begin{tabular}{|l|l|l|l|l|l|}
\hline Algorithm & Type & $\mathrm{N}$ & $\mathrm{K}$ & RMSE $_{\text {train }}$ & RMSE $_{\text {test }}$ \\
\hline RIT2NFS-WB & Type-2 & 200 & 7 & 0.0021 & 0.0021 \\
SEIT2FNN & Type-2 & 200 & 7 & 0.0022 & 0.0022 \\
McIT2FIS-US & Type-2 & 50000 & 5 & 0.0006 & 0.0006 \\
McFIS & Type-1 & 50000 & 20 & 0.0023 & 0.0023 \\
eTS & Type-1 & 50000 & 19 & - & 0.0080 \\
SAFIS & Type-1 & 50000 & 8 & - & 0.0112 \\
SONFIN & Type-1 & 50000 & 10 & - & 0.0130 \\
FMNN-FIS & Type-1 & 200 & 15 & 0.0024 & 0.0023 \\
FMNN-FIS & Type-1 & 50000 & 16 & 0.0013 & 0.0013 \\
\hline CFMN-FIS $^{\mathrm{a}}$ & Type-1 & 200 & 6 & 0.0015 & 0.0015 \\
CFMN-FIS $^{\mathrm{b}}$ & & 200 & 11 & 0.0007 & 0.0008 \\
CFMN-FIS $^{\mathrm{c}}$ & & 200 & 25 & 0.0005 & 0.0005 \\
CFMN-FIS $^{\mathrm{a}}$ & & 50000 & 6 & 0.0009 & 0.0009 \\
CFMN-FIS $^{\mathrm{b}}$ & & 50000 & 11 & 0.0001 & 0.0001 \\
CFMN-FIS $^{\mathrm{c}}$ & & 50000 & 25 & 0.00008 & 0.00008 \\
\hline
\end{tabular}

Table 2. Performances of various fuzzy models for mackeyglass of Example 4. $K$ represents the number of rules. RBF1 and RBF2 are two RBF networks.

\begin{tabular}{|l|l|l|l|}
\hline Algorithm & $\mathrm{K}$ & RMSE $_{\text {train }}$ & RMSE $_{\text {test }}$ \\
\hline RBF1 & - & - & 0.0013 \\
RBF2 & - & 0.0012 & 0.0013 \\
ANFIS & 16 & 0.0016 & 0.0015 \\
GEFREX & 20 & 0.0054 & 0.0061 \\
RBF-AFS & 23 & 0.0096 & 0.0114 \\
FMNN-FIS & 47 & 0.0036 & 0.0037 \\
\hline CFMN-FIS $^{\mathrm{a}}$ & 36 & 0.0016 & 0.0015 \\
CFMN-FIS $^{\mathrm{b}}$ & 52 & 0.0012 & 0.0011 \\
CFMN-FIS $^{\mathrm{c}}$ & 72 & 0.0010 & 0.0009 \\
\hline
\end{tabular}

\subsection{Example 5-Identification Performances on Three Real-World Data Sets}

In this example, the identification performance of CFMN-FIS is tested on three real-world data sets widely used in the fuzzy modeling literature. These data sets are neither arranged in generating order, nor in bunch of clusters. The purpose of this example is to show that CFMN-FIS is still able to learn the underlying system even when the input patterns are shuffled.

The first is the Box-Jenkins furnace data set ${ }^{35}$. It consists of 296 data points $[u(t), y(t)]$, from $t=1$ to 296, where $u(t)$ is the input gas flow rate and $y(t)$ is the output $\mathrm{CO}_{2}$ concentration. The system is modeled by $\widehat{y}(t)=f(u(t-4), y(t-1))$ and all the samples are used for training as done in Ref. 27. The parameters of CFMN-FIS on this data set are $[\theta=0.17$, $\phi=0.96, \Delta=1]$. FMNN-FIS is run with $\theta=0.2$.

The second is the abalone data set collected from the UCI machine learning repository. It consists of 4177 samples. The data are generated with random sampling, with 3342 samples for training and the remaining 835 samples for testing. The features length, diameter, height, whole weight, shucked weight, viscera weight, and shell weight are taken as input, the number of rings is taken as output as done in Ref. 26. The parameters of CFMN-FIS on this data set are $[\theta=0.6, \phi=0.97, \Delta=1]$. FMNNFIS is run with $\theta=0.47$.

The third is the auto MPG data set collected from the UCI machine learning repository. It consists of 
392 samples, out of which 272 samples are randomly chosen for training and the remaining 120 samples for testing. The three input features weight, acceleration, and model year are used for estimating the fuel consumption as done in Ref. 27. The parameters of CFMN-FIS on this data set are $[\theta=0.5, \phi=$ $0.92, \Delta=1.5]$. FMNN-FIS is tried with several $\theta \mathrm{s}$, but it's difficult to get the cluster number that is less than 10 on this dataset.

The results of CFMN-FIS and various algorithms are shown in Table 3, Table 4, and Table 5. The results of SEIT2FNN ${ }^{36}$, McIT2FIS-US ${ }^{27}$, and RIT2NFS-WB ${ }^{26}$ are adapted from $^{27}$. The performances of CFMN-FIS and FMNN-FIS on these data sets are comparable to those three Type-2 fuzzy systems. Note that both FMNN-FIS and CFMNFIS have the order-dependence feature, and Type2 fuzzy systems are generally considered to be better than Type-1 fuzzy systems ${ }^{37}$. Compared with FMNN, CFMN explicitly exploits the orderdependent information of data to ensure that estimated cluster centers are around the real ones even if the algorithm fails to estimate the true number of clusters. This order-dependence feature makes the generated fuzzy rules easier to cover the input space (see Fig. 12 for example), and also more meaningful for the FIS. Please note that the cluster number of CFMN in this example is also more controllable than FMNN, and this is attributed to introduction of the CF strategy which limits the algorithm's plasticity. More specifically, CFMN offers the user more flexibility of choosing parameters than FMNN.

Table 3. Performances of various fuzzy models for the furnace data set. $K$ represents the number of rules.

\begin{tabular}{|l|l|l|}
\hline Algorithm & K & RMSE \\
\hline SEIT2FNN & 19 & 0.2690 \\
McIT2FIS-US & 6 & 0.3181 \\
RIT2NFS-WB & 18 & 0.3527 \\
FMNN-FIS & 15 & 0.3472 \\
CFMN-FIS & 14 & 0.3385 \\
\hline
\end{tabular}

Table 4. Performances of various fuzzy models for the abalone data set. $K$ represents the number of rules.

\begin{tabular}{|l|l|l|l|}
\hline Algorithm & $\mathrm{K}$ & RMSE $_{\text {train }}$ & RMSE $_{\text {test }}$ \\
\hline McIT2FIS-US & 5 & 2.3357 & 1.8387 \\
SEIT2FNN & 5 & 2.3388 & 2.4330 \\
RIT2NFS-WB & 5 & 2.4047 & 2.1346 \\
FMNN-FIS & 4 & 2.0947 & 2.12299 \\
CFMN-FIS & 4 & 2.0989 & 2.1297 \\
\hline
\end{tabular}

Table 5. Performances of various fuzzy models for the auto MPG data set. $K$ represents the number of rules.

\begin{tabular}{|l|l|l|l|}
\hline Algorithm & $\mathrm{K}$ & RMSE $_{\text {train }}$ & RMSE $_{\text {test }}$ \\
\hline McIT2FIS-US & 3 & - & 2.6770 \\
RIT2NFS-WB & 4 & - & 2.7807 \\
SEIT2FNN & 4 & - & 2.7895 \\
CFMN-FIS & 3 & 2.7142 & 2.7402 \\
\hline
\end{tabular}

\section{Conclusions}

This study addresses the construction of TakagiSugeno-Kang (TSK) fuzzy models by means of clustering. We recast FMNN in the framework of ART and analyze the stability of FMNN. Then CFMN is developed to exploit the order-dependent information in the clustering process and to have enhanced ability of noise reduction and outlier detection. Owing to the introduction of contributionfactor (CF) strategy and the parameter $\Delta, \mathrm{CFMN}$ allows the user to have more control over the clustering process, so it is more intuitive in parameter choosing. The $\mathrm{CF}$ is also known as the typical pattern, and parameter $\phi$ specifies the membership threshold above which a pattern can be a CF of a cluster. CFMN is designed to have two operation modes. The normal mode is used when the data set is provided in sequence. Otherwise, the relearning mode is used. The stability issue is addressed and unnecessary overlaps in FMNN can be avoided in CFMN. Furthermore, CFMN can put cluster centers exactly in high-density regions without influence of low-density regions. This order-dependent feature ensures that estimated cluster centers are around the real ones even if the algorithm fails to estimate the true number of clusters and also allows CFMN to generate more meaningful fuzzy rules for the TSKtype CFMN-FIS. The effectiveness of CFMN and 
CFMN-FIS has been proven on the real-world and simulation data sets.

\section{Acknowledgements}

We thank the reviewers for their valuable comments. This work was supported by Projects in the National Science \& Technology Pillar Program during the Twelfth Five-Year Plan Period (Grant No.2014BAL05B02). This work was also supported by the National Natural Science Foundation of China (Grant No. 61773008).

\section{References}

1. T. Takagi, M. Sugeno, Fuzzy identification of systems and its applications to modeling and control, IEEE Transactions on Systems, Man, and Cybernetics SMC-15 (1) (1985) 116-132. http://dx.doi.org/10.1109/TSMC.1985.6313399 doi:10.1109/TSMC.1985.6313399.

2. J. Yen, L. Wang, C. W. Gillespie, Improving the interpretability of TSK fuzzy models by combining global learning and local learning, Fuzzy Systems, IEEE Transactions on 6 (4) (1998) 530-537.

3. A. K. Jain, Data clustering: 50 years beyond K-means, Pattern recognition letters 31 (8) (2010) 651-666.

4. S. L. Chiu, Fuzzy model identification based on cluster estimation., Journal of intelligent and Fuzzy systems 2 (3) (1994) 267-278, 02494.

5. E. Kim, M. Park, S. Ji, M. Park, A new approach to fuzzy modeling, Fuzzy Systems, IEEE Transactions on 5 (3) (1997) 328-337.

6. T. Pal, N. R. Pal, SOGARG: A self-organized genetic algorithm-based rule generation scheme for fuzzy controllers, IEEE Transactions on Evolutionary Computation 7 (4) (2003) 397415. http://dx.doi.org/10.1109/TEVC.2003.815377 doi:10.1109/TEVC.2003.815377.

7. D. Simon, Training fuzzy systems with the extended Kalman filter, Fuzzy Sets and Systems 132 (2) (2002) 189-199. http://dx.doi.org/10.1016/S01650114(01)00241-X doi:10.1016/S01650114(01)00241-X.

8. M. Delgado, A. Gómez-Skarmeta, F. Martin, A fuzzy clustering-based rapid prototyping for fuzzy rule-based modeling, IEEE Transactions on Fuzzy Systems 5 (2) (1997) 223-233. http://dx.doi.org/10.1109/91.580797 doi:10.1109/91.580797.

9. J. Abonyi, R. Babuska, F. Szeifert, Modified Gath-Geva fuzzy clustering for identification of Takagi-Sugeno fuzzy models, IEEE Transactions on Systems, Man, and Cybernetics, Part B (Cybernetics) 32 (5) (2002) 612-621. http://dx.doi.org/10.1109/TSMCB.2002.1033180 doi:10.1109/TSMCB.2002.1033180.

10. M. Kumar, R. Stoll, N. Stoll, A min-max approach to fuzzy clustering, estimation, and identification, IEEE Transactions on Fuzzy Systems 14 (2) (2006) 248262. http://dx.doi.org/10.1109/TFUZZ.2005.864081 doi:10.1109/TFUZZ.2005.864081.

11. G. Tsekouras, H. Sarimveis, E. Kavakli, G. Bafas, A hierarchical fuzzy-clustering approach to fuzzy modeling, Fuzzy Sets and

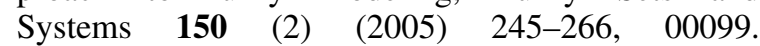
http://dx.doi.org/10.1016/j.fss.2004.04.013 doi:10.1016/j.fss.2004.04.013.

12. P. K. Simpson, Fuzzy min-max neural networks-Part 2: Clustering., IEEE Transactions on Fuzzy systems 1 (1) (1993) 32.

13. B. Gabrys, A. Bargiela, General fuzzy min-max neural network for clustering and classification, Neural Networks, IEEE Transactions on 11 (3) (2000) 769-783.

14. S. Grossberg, Adaptive pattern classification and universal recoding: II. Feedback, expectation, olfaction, illusions, Biological cybernetics 23 (4) (1976) 187202.

15. A.-H. T. Ji He, Modified ART 2A Growing Network Capable of Generating a Fixed Number of Nodes, IEEE transactions on neural networks / a publication of the IEEE Neural Networks Council 15 (3) (2004) 728-37. http://dx.doi.org/10.1109/TNN.2004.826220 doi:10.1109/TNN.2004.826220.

16. K.-L. Du, Clustering: A neural network approach, Neural Networks 23 (1) (2010) 89-107.

17. G. A. Carpenter, S. Grossberg, A massively parallel architecture for a self-organizing neural pattern recognition machine, Computer vision, graphics, and image processing 37 (1) (1987) 54-115.

18. G. A. Carpenter, S. Grossberg, D. B. Rosen, ART 2A: An adaptive resonance algorithm for rapid category learning and recognition, Neural networks 4 (4) (1991) 493-504.

19. B. Moore, ART 1 and pattern clustering, in: Proceedings of the 1988 Connectionist Models Summer School, Morgan Kaufmann Publishers San Mateo, CA, 1989, pp. 174-185.

20. H. Zhang, J. Liu, D. Ma, Z. Wang, Data-core-based fuzzy min-max neural network for pattern classification, Neural Networks, IEEE Transactions on 22 (12) (2011) 2339-2352.

21. R. Palaniappan, C. Eswaran, Using genetic algorithm to select the presentation order of training patterns that improves simplified fuzzy ARTMAP classification performance, Applied Soft Computing 9 (1) (2009) 100-106. 
22. M. Keyarsalan, G. A. Montazer, K. Kazemi, Font-based persian character recognition using Simplified Fuzzy ARTMAP neural network improved by fuzzy sets and Particle Swarm Optimization, in: 2009 IEEE Congress on Evolutionary Computation, 2009, pp. 3003-3009. http://dx.doi.org/10.1109/CEC.2009.4983322 doi:10.1109/CEC.2009.4983322.

23. C. M. Bishop, Pattern Recognition and Machine Learning, (Springer, 2006).

24. E. H. Ruspini, Numerical methods for fuzzy clustering, Information Sciences 2 (3) (1970) 319-350.

25. C.-F. Juang, C.-T. Lin, An online self-constructing neural fuzzy inference network and its applications, Fuzzy Systems, IEEE Transactions on 6 (1) (1998) 12-32.

26. C.-F. Juang, K.-J. Juang, Reduced interval type-2 neural fuzzy system using weighted bound-set boundary operation for computation speedup and chip implementation, Fuzzy Systems, IEEE Transactions on 21 (3) (2013) 477-491.

27. A. Das, K. Subramanian, S. Sundaram, An Evolving Interval Type-2 Neurofuzzy Inference System and Its Metacognitive Sequential Learning Algorithm, IEEE Transactions on Fuzzy Systems 23 (6) (2015) 2080-2093. http://dx.doi.org/10.1109/TFUZZ.2015.2403793 doi:10.1109/TFUZZ.2015.2403793.

28. H.-J. Rong, N. Sundararajan, G.-B. Huang, P. Saratchandran, Sequential adaptive fuzzy inference system (SAFIS) for nonlinear system identification and prediction, Fuzzy sets and systems 157 (9) (2006) 1260-1275.

29. K. Subramanian, S. Suresh, A meta-cognitive sequential learning algorithm for neuro-fuzzy inference system, Applied Soft Computing 12 (11) (2012) 36033614. http://dx.doi.org/10.1016/j.asoc.2012.06.012 doi:10.1016/j.asoc.2012.06.012.

30. J.-S. R. Jang, ANFIS: Adaptive-network-based fuzzy inference system, Systems, Man and Cybernetics, IEEE Transactions on 23 (3) (1993) 665-685.

31. M. Russo, Genetic fuzzy learning, IEEE Transactions on Evolutionary Computation 4 (3) (2000) 259-273. http://dx.doi.org/10.1109/4235.873236 doi:10.1109/4235.873236.

32. C. Harpham, C. W. Dawson, The effect of different basis functions on a radial basis function network for time series prediction: A comparative study, Neurocomputing 69 (16-18) (2006) 2161-2170. http://dx.doi.org/10.1016/j.neucom.2005.07.010 doi:10.1016/j.neucom.2005.07.010.

33. H. Du, N. Zhang, Time series prediction using evolving radial basis function networks with new encoding scheme, Neu-

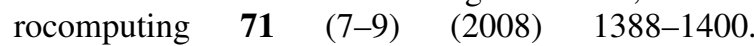
http://dx.doi.org/10.1016/j.neucom.2007.06.004 doi:10.1016/j.neucom.2007.06.004.

34. K. B. Cho, B. H. Wang, Radial basis function based adaptive fuzzy systems and their applications to system identification and prediction, Fuzzy Sets and Systems 83 (3) (1996) 325339. http://dx.doi.org/10.1016/0165-0114(95)003223 doi:10.1016/0165-0114(95)00322-3.

35. G. E. Box, G. M. Jenkins, G. C. Reinsel, G. M. Ljung, Time Series Analysis: Forecasting and Control, (John Wiley \& Sons, 2015).

36. C.-F. Juang, Y.-W. Tsao, A Self-Evolving Interval Type-2 Fuzzy Neural Network With Online Structure and Parameter Learning, IEEE Transactions on Fuzzy Systems 16 (6) (2008) 14111424. http://dx.doi.org/10.1109/TFUZZ.2008.925907 doi:10.1109/TFUZZ.2008.925907.

37. J. M. Mendel, Explaining the Performance Potential of Rule-Based Fuzzy Systems as a Greater Sculpting of the State Space, IEEE Transactions on Fuzzy Systems, vol. PP, no. 99, pp. 1-1, 2017. 